14

\title{
Изучение быстрых релаксаций в стеклообразном полимере методом длинноволновой ИК-спектроскопии
}

\author{
(C) В.А. Рыжов \\ Физико-технический институт им. А.Ф. Иофрфе РАН, \\ Санкт-Петербург, Россия \\ E-mail: v.ryzhov@mail.ioffe.ru
}

(Поступила в Редакцию 12 декабря 2016 г.)

Изучена температурная зависимость длинноволновых ИК-спектров стеклообразного полимера полиметилметакрилата в диапазоне $v=10-130 \mathrm{~cm}^{-1}$ при температурах от 90 до $420 \mathrm{~K}$. Их анализ позволил обнаружить поглощение, соответствующее двум быстрым релаксационным процессам: 1) $180^{\circ}$ поворотам ОСО-плоскости боковых групп; 2) переориентации звеньев основной цепи. Найдены энергии активации этих процессов.

DOI: 10.21883/FTT.2017.07.44609.440

\section{1. Введение}

Разработке молекулярных механизмов сегментальной динамики в полимерах посвящено большое число теоретических и экспериментальных исследований, приведших к пониманию природы основных релаксационных переходов. В то же время вопросы, касающиеся более локальной, мелкомасштабной (в пределах боковой группы или звена цепи) подвижности, нуждаются в дальнейшем выяснении, тем более что макро- и микродинамика в полимерах тесно взаимосвязаны. Отметим, что основной метод исследования локальной динамики полимерных молекул - молекулярно-динамическое моделирование - хотя и является весьма информативным, все же определяет не все детали динамики.

Сравнительно недавно к исследованию процессов более быстрых, чем сегментальные перегруппировки в макромолекулах, стала привлекаться рамановская спектроскопия, показавшая возможность еднообразного описания как индивидуальной, так и коллективной подвижности молекул [1]. Важным явилось установление того факта, что в рамановских спектрах полимеров в диапазоне $0.03-3 \mathrm{THz}\left(\sim 1-100 \mathrm{~cm}^{-1}\right)$ проявляются как крутильное колебание отдельных звеньев (либрационная полоса), так и их коллективное движение („бозонный“ пик), а также релаксационная составляющая (, $\beta$-fast“ потери) на частотах квазиупругого рассеяния [2,3].

Природа быстрых молекулярных процессов, наблюдаемых в низкочастотных спектрах, в настоящее время активно обсуждается в литературе, в том числе на основе данных, полученных методом длинноволновой ИК (FIR)-спектроскопии, позволяющей конкретизировать их механизмы $[4,5]$. В модели связанных мод теории стеклования эти процессы относят к проявлению $\beta$-релаксации, предшествующей релаксации структурной [6]. Рассматривается и вклад элементарных процессов типа локальных конформационных переориентаций в основной цепи и боковых группах [7]. Подобная неоднозначность отнесения быстрых процессов в полимерах объясняется тем, что зачастую оно ведется с использованием феноменологических представлений, основанных на таких усредненных характеристиках вещества, как свободный объем, коэффициенты вязкости, диффузии и т.п.

Более уверенно конкретизировать механизмы молекулярных движений, ответственных за пикосекундную динамику молекул, позволяют молекулярно-кинетические теории, основанные на представлениях о генетической связи колебательных и релаксационных форм движения [8]. Примером такого подхода может служить работа [9], в которой было установлено, что релаксационная составляющая на частотах квазиупругого рассеяния в рамановских спектрах полистирола и поликарбоната, измеренных в интервале 3-1000 GHz при температурах ниже температуры стеклования, обусловлена температурно-активированными переходами через барьер высотой $\sim 500 \mathrm{~K}[10]$.

Возможность определить величину энергии активации быстрых перегруппировок в полиметилметакрилате (РММА) на основе изменений спектральных параметров низкочастотного поглощения с температурой использовалась и в настоящей работе для выяснения природы релаксационного вклада в FIR-спектры этого полимера.

\section{2. Методика}

Опыты проводились на пленках блочного атактического полиметилметакрилата среднечисловым молекулярным весом $M_{n} \approx 500000 \mathrm{~g} / \mathrm{mol}$ и толщиной $\sim 100 \mu \mathrm{m}$, полученных прессованием при $150^{\circ} \mathrm{C}$ и выдержкой в течение $3-5 \mathrm{~h}$ при $110^{\circ} \mathrm{C}$. FIR-спектры регистрировались на двух спектрометрах: в диапазоне $10-50 \mathrm{~cm}^{-1}$ на спектрометре ЛГУ [11], модернизированном с помощью приемника ОАП-7 и новой системы фильтрации; в диапазоне $50-150 \mathrm{~cm}^{-1}$ на спектрометре FIS-21 Hitachi. Спектры записывались с разрешением $1-2 \mathrm{~cm}^{-1}$ 
при отношении сигнал/шум порядка 100. Погрешность измерения частоты составляла $2-3 \mathrm{~cm}^{-1}$, погрешность измерения коэффициента поглощения $k=(t)^{-1} \ln \left(I_{0} / I\right)$ (где $I_{0}$ и $I-$ интенсивности падающего и прошедшего через образец толщиной $t$ излучения соответственно) составляла $5-10 \%$. Для измерения спектров в температурном интервале от 90 до $273 \mathrm{~K}$ использовалась кювета-криостат с окнами из кристаллического кварца. FIR-спектры в интервале от комнатной температуры до $\sim 413 \mathrm{~K}$ получали, используя термостатированную кювету с сапфировыми окнами. Теплоизоляция в обоих случаях достигалась размещением кювет в вакууме. Температура образца измерялась термопарой медь-константан, ее стабильность была не ниже $\pm 2^{\circ} \mathrm{C}$. В интервале от 10 до $50 \mathrm{~cm}^{-1}$ для каждой температуры проводилось до пяти параллельных измерений.

\section{3. Результаты и их обсуждение}

FIR-спектры PMMA представлены на рис. 1 в виде зависимости $k / v$ от $v$. Представление FIR-спектров в таких координатах позволяет сравнивать их с paмановскими спектрами, приведенными в координатах $I(v) /(N+1)$, где $I(v)$ - измеренная интенсивность рассеяния, а $N+1$ - Бозе-фактор.

Как и приведенные рамановские спектры РММА, зарегистрированные в области $5-150 \mathrm{~cm}^{-1}$ для разных температур [12], приведенные FIR-спектры PMMA имеют интенсивную полосу при $\sim 90 \mathrm{~cm}^{-1}$ с особенностью на частотах, близких к положению бозонного пика (BP) в рамановских спектрах.

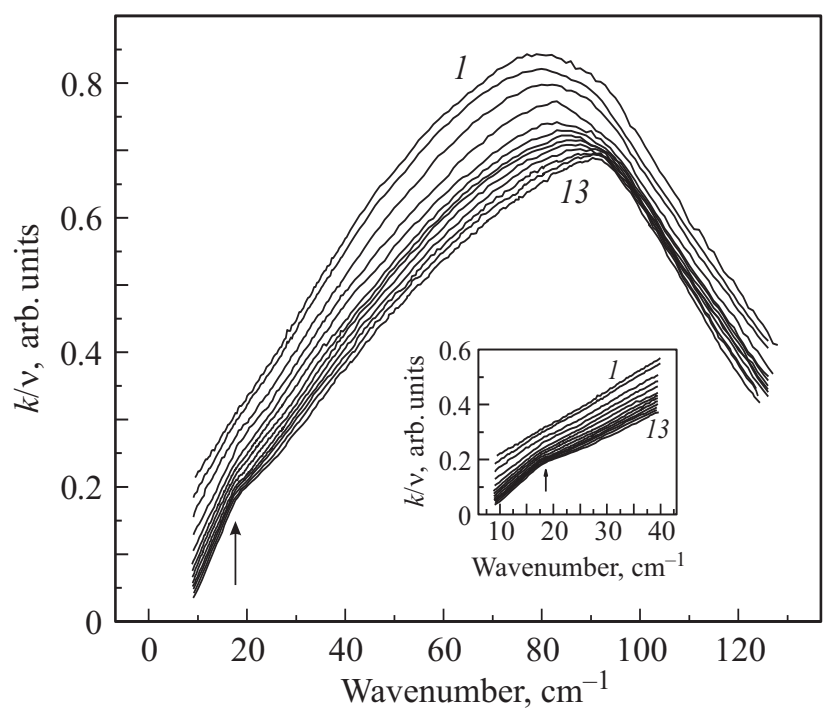

Pис. 1. Приведенные FIR-спектры ПMМA в области $10-130 \mathrm{~cm}^{-1}$ при различных температурах. $T, \mathrm{~K}: 1-420$, $2-403,3-368,4-333,5-293,6-273,7-253$, $8-233,9-193,10-173,11-153,12-123,13-90$. На вставке показаны начальные участки кривых в увеличенном масштабе, стрелки отмечают положение бозонного пика в спектрах.

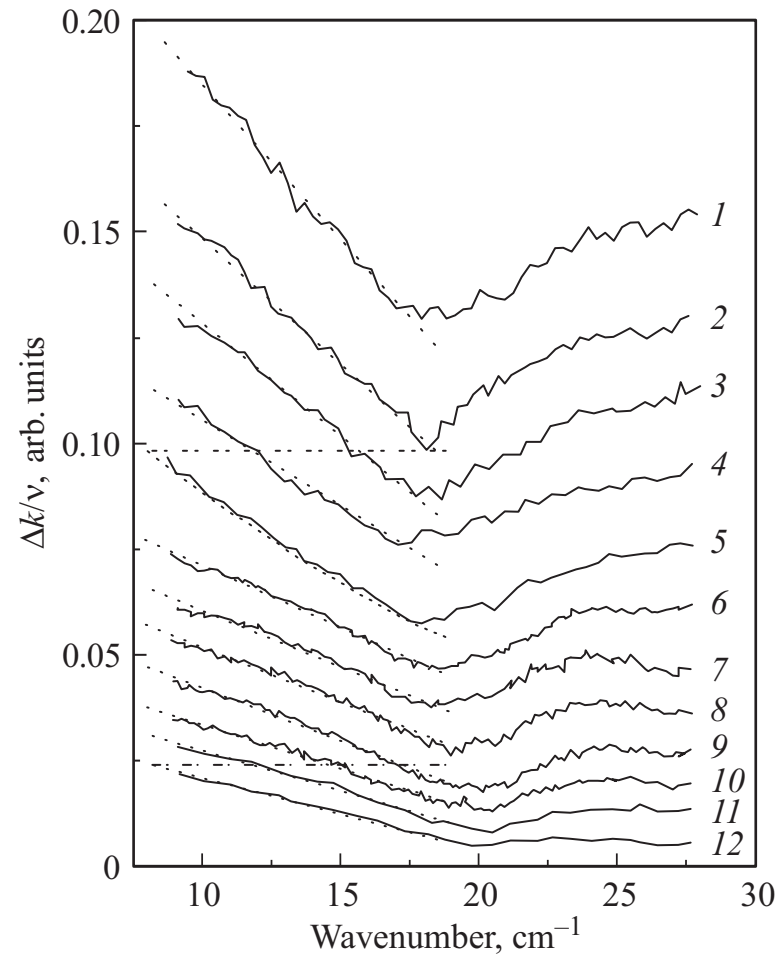

Pис. 2. Спектры, полученные вычитанием из FIR-спектров PMМА при температурах от 420 до $123 \mathrm{~K}$ спектра PMМА при $90 \mathrm{~K}$. Обозначения те же, что на рис. 1.

Из рис. 1 видно, что по мере повышения температуры полоса поглощения при $\sim 90 \mathrm{~cm}^{-1}$ несколько уширяется и ее интенсивность растет, а максимум сдвигается на $3-5 \mathrm{~cm}^{-1}$ к низким частотам. Последнее обусловлено, очевидно, увеличением амплитуды и ангармоничности колебаний из-за ослабления межмолекулярных взаимодействий. Особый интерес представляет рост интенсивности ее длинноволнового крыла на частотах ниже $\mathrm{BP}$, что свидетельствует об увеличении релаксационной составляющей спектра [12]. Динамику роста интенсивности длинноволнового крыла полосы иллюстрирует рис. 2, на котором приведены разностные спектры, полученные вычитанием из них спектра РММА при $90 \mathrm{~K}$, представляющего собой, как это следует из работы [12], в основном чисто колебательный вклад.

Как видно из зависимости интенсивности разностного спектра от температуры (рис. 3), рост поглощения в области $8-20 \mathrm{~cm}^{-1}$ не является монотонным. В ней явно прослеживаются две стадии: „низкотемпературная“" (от 90 до $\sim 260 \mathrm{~K}$, и более резкая „высокотемпературная“ (от $\sim 260$ до $420 \mathrm{~K}$ ). Поскольку температура, с которой начинается конформационная подвижность в РММА, близка к $260 \mathrm{~K}$ [13], это позволяет предположить, что резкий рост интенсивности разностного спектра обусловлен именно таким процессом.

Чтобы подтвердить это предположение, оценим высоту потенциального барьера „высокотемпературного“ релаксационного процесса, используя имеющиеся экспе- 


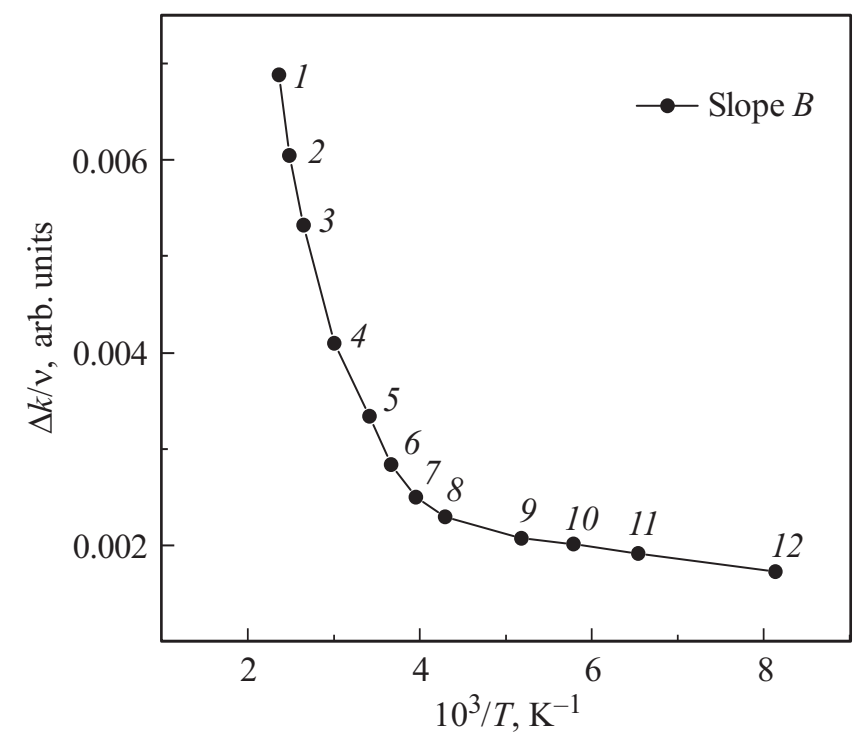

Pис. 3. Температурная зависимость интенсивности FIR-спектров РММА в области $8-20 \mathrm{~cm}^{-1}$. Обозначения те же, что на рис. 1.

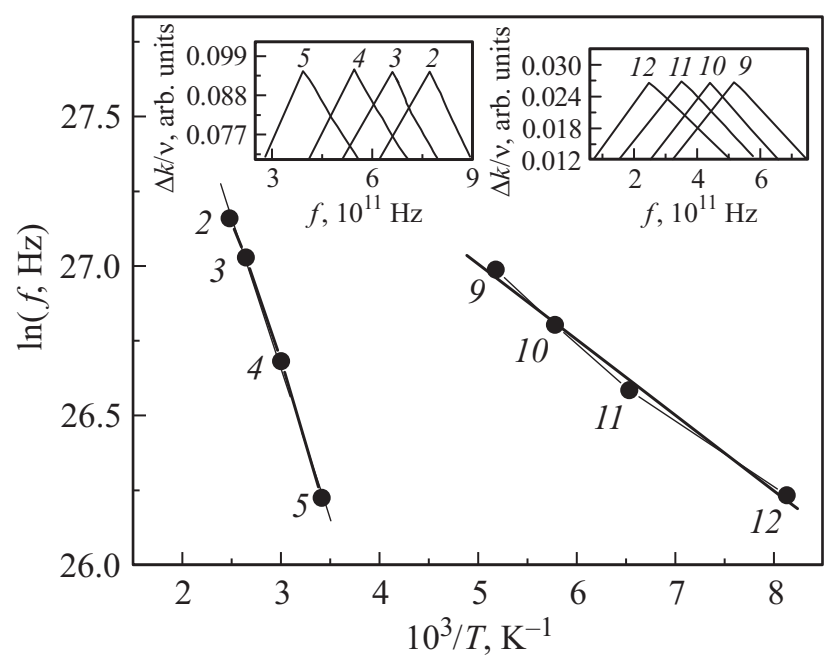

Рис. 4. Зависимости частотного положения „высокотемпературных“ $(2-5)$ и „низкотемпературных“ $(9-12)$ релаксационных потерь от температуры. На вставках схематически показано смещение пика этих потерь с температурой. Обозначения те же, что на рис. 1.

риментальные данные по его температурному смещению. Например, так, как это делается в методике динамического механического анализа, дифференциальной сканирующей калориметрии или при диэлектрических измерениях, когда энергию активации релаксационного процесса определяют по линейной зависимости смещения частоты релаксационного пика $f$ с температурой $\ln f(1 / T)$ и формуле $E=-R d(\ln f) / d(1 / T)$, где $R$ - универсальная газовая постоянная, а $f=c v$ ( $c$ - скорость света в вакууме).
Поскольку на высоких частотах релаксационный пик с температурой сдвигается параллельно самому себе, сохраняя амплитуду и полуширину [14], построение такой зависимости можно проводить не только по положению максимума. В нашем случае пик релаксационных потерь не попадает в исследованный интервал длин волн, поэтому смещение его частоты определялось по смещению низкочастотного крыла разностного спектра при вариации температуры. На рис. 2 изменение положения низкочастотного крыла при $\Delta k / v=0.09$ для „высокотемпературного“ и при $\Delta k / v=0.02$ для „низкотемпературного“ релаксационных процессов показаны горизонтальными штриховыми линиями.

Полученные таким образом зависимости $\ln f(1 / T)$ представлены на рис. 4 (вставки иллюстрируют смещение „пиков“ выделенных релаксационных процессов). По наклону этих зависимостей $(B)$ и формуле $E=-8.3 B(\mathrm{~J} / \mathrm{mol})$ были определены энергии активации „низкотемпературного“ и „высокотемпературного“ процессов, составившие $\sim 2$ и $8 \mathrm{~kJ} / \mathrm{mol}$ соответственно.

Энергия активации первого из них близка к энергии активации быстрой релаксации в поливинилацетате $(1.9 \mathrm{~kJ} / \mathrm{mol})$, отнесенной к $180^{\circ}$ поворотам ОСО-группы [15]. Барьер второго быстрого процесса соответствует разности энергий поворотных изомеров в PMMA, а то, что он начинает проявляться при температуре появления конформационной подвижности в PMМА, позволяет считать его высокочастотным аналогом $\beta$-релаксации в этом полимере.

\section{4. Заключение}

Таким образом, проведенное в работе изучение FIR-спектров поглощения полиметилметакрилата в диапазоне $v=10-130 \mathrm{~cm}^{-1}$ при температурах от 90 до $420 \mathrm{~K}$, показало, что они содержат информацию не только о локальной колебательной подвижности мономерных звеньев этого стеклообразного полимера, но и о том, что начиная с температуры $\beta$-перехода $T \approx 260 \mathrm{~K}$ в РММА реализуется релаксационная динамика.

По линейной зависимости частотного положения релаксационных потерь с температурой найдены энергии активации двух быстрых релаксационных процессов в этом стеклообразном полимере: $180^{\circ}$ поворотов ОСО-плоскости боковых групп и конформационной переориентации мономерных звеньев.

\section{Список литературы}

[1] У. Коффи, М. Ивенс, П. Григолини. Молекулярная диффузия и спектры. Наука, М. (1987). 397 с.

[2] В.К. Малиновский, В.Н. Новиков, А.П. Соколов. ФХС 22, 204 (1996).

[3] G.P. Johari. J. Non-Cryst. Solids. 307-310, 114 (2002).

[4] V.A. Bershtein, V.A. Ryzhov. Adv. Polym. Sci. 114, 43 (1994).

[5] В.А. Рыжов. ФТТ 44, 2229 (2002).

[6] W. Götze, L. Sjogren. Rep. Prog. Phys. 55, 241 (1992). 
[7] T. Kanaya, K. Kaji. Adv. Polym. Sci. 154, 87 (2001).

[8] Ю.Я. Готлиб, А.А. Даринский, Ю.Е. Светлов. Физическая кинетика макромолекул. Химия, Л. (1986). 272 с.

[9] N.V. Surovtsev, J.A. Wiedersich, V.N. Novikov, E. Rossler, A.P. Sokolov. Phys. Rev. B 58, 14888 (1998).

[10] K.S. Gilroy, W.A. Phillips. Phil. Mag. 43, 735 (1981).

[11] В.А. Рыжов, М.В. Тонков. В сб.: Молекулярная спектроскопия / Под ред. М.О. Буланина. Изд-во ЛГУ, Л. (1973). C. 103.

[12] N.V. Surovtsev, T. Achibat, E. Duval, A. Mermet, V.N. Novikov. J. Phys: Condens. Matter. 7, 8077 (1997).

[13] В.А. Берштейн, В.М. Егоров. Дифференциальная сканирующая калориметрия. Химия, Л. (1990). 255 р.

[14] H.Z. Cummins, G. Li, Y.H. Hwang, G.Q. Shen, W.M. Du, J. Hernandez, N.J. Tao. Z. Phys. B 103, 501 (1997).

[15] C. Zhang, V. Arrighi, S. Gagliardi, I.J. McEwen, J. Tanchawanich, M.T.F. Telling, J.-M. Zanotti. Chem. Phys. 328, 53 (2006). 